\title{
The chitobiose-binding protein, DasA, acts as a link between chitin utilization and morphogenesis in Streptomyces coelicolor
}

\author{
Séverine Colson, ${ }^{1}$ Gilles P. van Wezel, ${ }^{2}$ Matthias Craig, ${ }^{1}$ Elke E. E. Noens, ${ }^{2}$ \\ Harald Nothaft, ${ }^{3}$ A. Mieke Mommaas, ${ }^{4}$ Fritz Titgemeyer, ${ }^{3}$ \\ Bernard Joris ${ }^{1}$ and Sébastien Rigali ${ }^{1}$
${ }^{1}$ Centre d'Ingénierie des Protéines, Université de Liège, Institut de Chimie B6a, B-4000 Liège, Belgium
${ }^{2}$ Microbial Development, Leiden Institute of Chemistry, Leiden University, PO Box 9502, 2300 RA Leiden, The Netherlands 91058 Erlangen, Germany
${ }^{4}$ Center for Electron Microscopy, Leiden University Medical Center, Leiden, The Netherlands \\ ${ }^{3}$ Lehrstuhl für Mikrobiologie, Friedrich-Alexander-Universität Erlangen-Nürnberg, Staudtstrasse 5,
}

Correspondence

Sébastien Rigali

srigali@ulg.ac.be

\begin{abstract}
Streptomycetes are mycelial soil bacteria that undergo a developmental programme that leads to sporulating aerial hyphae. As soil-dwelling bacteria, streptomycetes rely primarily on natural polymers such as cellulose, xylan and chitin for the colonization of their environmental niche and therefore these polysaccharides may play a critical role in monitoring the global nutritional status of the environment. In this work we analysed the role of DasA, the sugar-binding component of the chitobiose ATP-binding cassette transport system, in informing the cell of environmental conditions, and its role in the onset of development and in ensuring correct sporulation. The chromosomal interruption of $d a s A$ resulted in a carbon-source-dependent vegetative arrest phenotype, and we identified a second DasR-dependent sugar transporter, in addition to the $N$-acetylglucosamine phosphotransferase system (PTS ${ }^{\text {GlcNAc }}$ ), that relates primary metabolism to development. Under conditions that allowed sporulation, highly aberrant spores with many prematurely produced germ tubes were observed. While GlcNAc locks streptomycetes in the vegetative state, a high extracellular concentration of the GlcNAc polymer chitin has no effect on development. The striking distinction is due to a difference in the transporters responsible for the import of GlcNAc, which enters via the PTS, and of chitin, which enters as the hydrolytic product chitobiose $\left(\mathrm{GlcNAc}_{2}\right)$ through the DasABC transporter. A model explaining the role of these two essentially different transport systems in the control of development is provided.
\end{abstract}

Received 7 August 2007

Revised 26 October 2007

Accepted 29 October 2007

\section{INTRODUCTION}

Streptomycetes are Gram-positive soil bacteria with a complex life cycle that starts with the germination of a single spore, which grows out to form a branched mycelium of vegetative hyphae. In adverse environmental conditions, morphological differentiation is initiated by a complex spatio-temporal programme by which aerial hyphae produce chains of hydrophobic spores. Mutant screens identified two classes of developmental mutants, namely those that are locked in the vegetative state and fail to produce aerial hyphae, the so-called bald (bld) mutants,

Abbreviations: ABC, ATP-binding cassette; PEP, phosphoenolpyruvate; PTS, phosphotransferase system.

A supplementary figure is available with the online version of this paper. and those that do produce aerial hyphae but fail to complete sporulation, which are called white (whi) mutants, reflecting the lack of grey-pigmented spores (Chater, 1972; Hopwood et al., 1970). Perhaps the most important signal for the onset of development is reduced nutrient availability (Chater \& Horinouchi, 2003), and this raises the question as to how these nutritional signals are sensed and transmitted to the cell to control the developmental programme. As soil-dwelling organisms, streptomycetes often use hydrolysis products of the abundant polysaccharides cellulose, xylan, and chitin as food sources. The first two are of plant cell-wall origin, and the third mainly originates from the cell walls of fungi and the cuticles of crustaceans or insects. Their presence is a sign of a rich environmental niche, and as abundant carbon sources they may play a critical role in monitoring the 
global nutritional status of the environment. We therefore postulated that their respective sensors/transporters might be at the extremity of transduction pathways that correlate primary metabolism to developmental genes. The hydrolysis of these polysaccharides leads to the direct derivative products cellobiose, xylobiose and chitobiose, which in turn are the best inducers of enzymes involved in their self production (Godden et al., 1989; Miyashita et al., 2000).

Recently, we showed that the addition of $\mathrm{N}$-acetylglucosamine (GlcNAc), the monomer unit of chitin, on rich medium (R2YE) was able to lock Streptomyces coelicolor in the vegetative growth phase and this molecule was therefore considered as a critical signal to the developmental programme (Rigali et al., 2006). GlcNAc is transported via the sugar phosphotranferase system (PTS) (Nothaft et al., 2003) and the five genes involved ( $p t s I$ for enzyme EI, $p t s H$ for $\mathrm{HPr}, \mathrm{crr}$ for enzyme EIIA, malX2 for enzyme EIIB ${ }^{\mathrm{GlcNAc}}$ and nagE2 for enzyme EIIC ${ }^{\mathrm{GlcNAc}}$ ) are transcriptionally repressed by the GntR-type regulator DasR (Rigali et al., 2002, 2004, 2006). Previous studies on developmental mutants of streptomycetes have led to the identification of four genes encoding members of the GntR family: whiH (Ryding et al., 1998), dasR (Rigali et al., 2006), SCO7168 (Hillerich \& Westpheling, 2006) and $\operatorname{devA}$ (Hoskisson et al., 2006), supporting the idea that members of this family are critical control points for development in these micro-organisms. The computational prediction of the DasR regulon in $S$. coelicolor suggests other DasR-dependent transporters, and we investigated the question if, besides the PTS ${ }^{\mathrm{GlcNAc}}$, other DasR-dependent transporters are also involved in the control of morphological differentiation of streptomycetes and if so, what the transported molecules would be.

In a previous communication we showed that DasR is a pleiotropic regulator that is essential for development of $S$. coelicolor (Rigali et al., 2006). After the PTS ${ }^{\mathrm{GlcNAc}}$, one of the known targets of DasR in S. coelicolor is a cluster of three genes that compose an ATP-binding cassette (ABC) sugar transporter, i.e. the DasABC system involved in chitobiose transport (Rigali et al., 2004; Seo et al., 2002). This system consists of dasA (SCO5232), encoding the chitobiose-binding protein, located upstream of das $B$ (SCO5233) and dasC (SCO5234), encoding the respective permeases. Downstream of dasC, SCO5235 (dasD) encodes an intracellular $\beta$-N-acetylglucosaminidase that could hydrolyse the transported chitobiose into the GlcNAc subunits. While the synteny strongly suggests that the SCO5231-SCO5234 genes of S. coelicolor correspond to the dasRABC gene cluster of Streptomyces griseus (Seo et al., 2002), the overall similarity of the primary sequences is very low, namely $33 \%$ for the solute-binding protein DasA, and $32 \%$ and $43 \%$ for the transmembrane proteins DasB and DasC, respectively, suggesting different transported sugars with therefore potentially different repercussions on morphogenesis. The gene encoding the solute-binding protein of $S$. griseus was previously shown to be involved in the control of morphogenesis, as its inactivation led to a bald phenotype (Seo et al., 2002).

In this work we show that the chitobiose-binding component DasA of $S$. coelicolor is also essential for development, thus uncovering another link between sugar metabolism and the start of the developmental programme. We also show that under conditions that allow sporulation by the mutant, highly aberrant spores are produced with many prematurely produced germ tubes, a sign that the role of DasA extends into the latest stages of sporulation. We also show that although GlcNAc (PTS transport-dependent) locks streptomycetes in the vegetative state, a high extracellular concentration of the GlcNAc-polymer chitin (DasABC transport-dependent) has no effect on streptomycete development. We provide a model explaining the role of these two essentially different transport systems in the control of development.

\section{METHODS}

Bacterial strains and culture conditions. All media and routine Streptomyces techniques are described in the Streptomyces manual (Kieser et al., 2000). Liquid cultures of S. coelicolor were grown at $28{ }^{\circ} \mathrm{C}$ using tryptic soy broth without $1 \%$ glycerol as complex medium (TSB, Difco) or minimal medium (MM). Soy flour mannitol (SFM) medium was used for making spore suspensions. R2YE agar plates were used for regeneration of protoplasts and, after addition of the appropriate antibiotic, for selecting recombinants. Phenotypic characterization of mutants was done on SFM, R2YE and MM agar plates with various carbon sources as indicated in the text. S. coelicolor strains are listed and referenced in Table 1. Others streptomycetes used in this study were Streptomyces antibioticus IMRU 3720, Streptomyces limosus ATCC 19778, Streptomyces roseosporus ATCC

Table 1. Strains of $S$. coelicolor used in this study

S. coelicolor M145 was the parental strain for the various mutants.

\begin{tabular}{|lll|}
\hline S. coelicolor strains & \multicolumn{1}{c|}{ Genotype } & \multicolumn{1}{c|}{ Reference } \\
\hline M145 & Parental strain, SCP1 ${ }^{-} \mathrm{SCP}^{-}$, prototroph & Kieser et al. $(2000)$ \\
BAP17, $\Delta g l c P 1$ & M145 glcP1:: hyg & van Wezel et al. $(2005)$ \\
BAP28, $\Delta$ chiSR & M145 chiS::aacC4::chiR & This work \\
BAP29, $\Delta$ dasR & M145 dasR::aacC4 & Rigali et al. $(2006)$ \\
SAF3, dasA mutant & M145 dasA::aacC4 & This work \\
M145 $\Delta g l k$ & M145 glk::aacC4 & This work \\
\hline
\end{tabular}


31568, Streptomyces acrimycini DSM 40540 and Streptomyces cacaoi KCC-S0352.

Construction of the $\operatorname{das} A, \boldsymbol{g l k}$, and chiSR mutants. The derivatives of cosmids SC7E4 and 6E10 carrying Tn5062 insertions in SCO5232 (dasA) and SCO2126 ( $g l k$ ) (obtained from Paul Dyson, Swansea, UK), generated using the in vitro transposition method of Bishop et al. (2004), were introduced into S. coelicolor M145 by conjugation from Escherichia coli ET12567/pUZ8002. Mutants exhibiting the double-crossover phenotype (apramycin-resistant, kanamycin-sensitive) were confirmed by PCR, and designated SAF3 and SAF5 for dasA and $g l k$, respectively. Verification of the correct recombination events was performed by PCR and Southern hybridization. Saito et al. (2007) reported that transcription of das $B C$ is independent of the transcription of das $A$, which ensures the absence of a polar effect on the das $B C$ genes. For complementation of the das $A$ mutant we used plasmid pSET151 harbouring the dasA gene and its promoter region (nt positions $-361 /+1278$ relative to the start of dasA). pSET151 lacks a Streptomyces origin of replication, and it can therefore be maintained only by integration into the genome. The selection marker of the plasmid is $t s r$ for thiostrepton resistance.

The chiSR mutant BAP28 (M145 chiS: : aacC4:: chiR) was created by replacing nucleotides 566-1142 inside the chiS gene by the apramycin resistance gene cassette, using pWHM3. This is a multicopy E. coliStreptomyces shuttle plasmid that is readily lost when antibiotic pressure (the plasmid harbours the tsr gene for thiostrepton resistance) is not maintained. The desired recombinants carry apramycin resistance, but are sensitive to thiostrepton due to loss of the vector sequences after double crossing over. Verification of the correct recombination events was performed by PCR.

Microscopy. Morphological studies of surface-grown aerial hyphae and spores of S. coelicolor M145 and SAF3 by cryo-scanning electron microscopy were performed using a JEOL JSM6700F scanning electron microscope. Transmission electron microscopy for analysis of thin sections of hyphae and spores was performed with a Philips EM410 transmission electron microscope. For stereomicroscopy we used an automated Zeiss Lumar V12 fluorescence microscope at 12$150 \times$ magnification, or an automated Leica MZ-16FA with up to $180 \times$ magnification and a compound lens for maximal $660 \times$ magnification, to establish the presence of aerial hyphae and spores. Phase-contrast microscopy was done with a Zeiss standard 25 microscope with 100-1000 $\times$ magnification. A 5-Mp CCD camera was used for photography. Images were acquired using the supplier's software and processed by Adobe Photoshop CS2 (Version 9.0).

RT-PCR. RNA was isolated from mycelium of S. coelicolor M145 and BAP29. Minimal medium plates containing $0.5 \%$ mannitol were inoculated with spores on cellophane discs and samples were collected after $30 \mathrm{~h}$ (vegetative growth), $42 \mathrm{~h}$ (initiation of aerial growth) and $72 \mathrm{~h}$ (aerial growth and spores) incubated at $28{ }^{\circ} \mathrm{C}$. RT-PCR analyses were conducted with the Superscript III One-Step RT-PCR kit (Invitrogen). RT-PCRs without reverse transcription were used as a control for absence of residual DNA. For semiquantitative analysis, samples were taken at four-cycle intervals between cycles 24 and 35 to compare non-saturated PCR product formation. Data were verified in three independent experiments. The oligonucleotides used to assess dasA transcription were $5^{\prime}$-CGACATGAAAGTGCAGGTGG-3' and $5^{\prime}$-TCGGGCTTGGTCAGCAAAAGC- $3^{\prime}$. rpsI was used as control ( $5^{\prime}$ GAGACCACTCCCGAGCAGCCGC-3' and 5'-GTAGCGGTTGTCCAGCTCGAGCA-3' ${ }^{\prime}$.

Enzyme assays. TSB ( $25 \mathrm{ml}$ volumes) supplemented with $1 \%(\mathrm{v} / \mathrm{v})$ glycerol was inoculated with either S. coelicolor M145 or SAF3 spore suspensions and cultures were grown to the exponential growth phase. Then $200 \mathrm{mg}$ fresh weight was used to inoculate $25 \mathrm{ml} \mathrm{MM}$ with various carbon sources $(1 \%, \mathrm{w} / \mathrm{v})$. Three samples were collected for all conditions after $48 \mathrm{~h}$ and mycelia and supernatants were separated. Mycelium was resuspended in water and disrupted by sonication. The BCA protein assay (Pierce) was used for determining protein concentrations. Chitinase activity measurements were performed as described by Zhang et al. (2002) using a colorimetric assay with carboxymethylchitin-Remazol Brilliant Violet 5R (Loewe Biochemica) as substrate. $\beta$-N-Acetylglucosaminidase activity measurements were performed at $37{ }^{\circ} \mathrm{C}$ as described by Kubota et al. (2004) using a colorimetric assay with $p$-nitrophenyl $N$-acetyl- $\beta$-Dglucosaminide (Sigma-Aldrich) as substrate. Results are expressed as a percentage of the activity measured for wild-type S. coelicolor M145 in induced culture conditions ( $1 \%$ chitin) and are the mean of three to six independent cultures.

\section{RESULTS}

\section{das $A$ null mutants have a highly aberrant phenotype}

To analyse the role of dasA in S. coelicolor, a dasA null mutant was generated from the parental strain S. coelicolor M145 using a derivative of cosmid SC7E4 carrying the Tn5062 transposon (conferring apramycin resistance) inserted into the dasA gene at nucleotide position 5692637 of the S. coelicolor chromosome (335 nt downstream of the translational start site of dasA). This cosmid derivative, generated using the in vitro transposition method of Bishop et al. (2004), was introduced into S. coelicolor by conjugation from E. coli. Mutants exhibiting the double-crossover phenotype (apramycin-resistant, kanamycin-sensitive) were confirmed by PCR, and one was designated SAF3. The resulting mutant has the egfp gene under the control of the dasA promoter (see below). Interestingly, the das A mutant showed medium-dependent development, only failing to produce aerial hyphae and spores on glucose-containing media (MM mannitol + glucose and R2YE agar plates; Fig. 1). Thus, dasA is classified as a novel bld mutant of $S$. coelicolor. Many of the bld mutants have a conditional phenotype, underlining the importance of sugar metabolism and carbon catabolite control in the control of development (Pope et al., 1996; Nodwell et al., 1999). Interestingly, the developmental deficiency was particularly pronounced in sections with high density, while separate colonies of SAF3 eventually produced aerial hyphae and spores at the colony edges (Fig. 1). This suggests that the developmental block is dependent on a quorum-sensing-like mechanism, and is relieved when colony density is lower.

Closer inspection by cryo-scanning electron microscopy showed a strictly bald phenotype when the dasA mutant was grown on R2YE agar plates, while the parent M145 produced normal aerial hyphae and spores (Fig. 2a, b). In contrast, on SFM agar plates the dasA mutant did produce aerial hyphae and spores, though sporulation was far less abundant than in the parent M145 (Fig. 2c, d). Excitingly, the dasA mutant had highly aberrantly shaped spores, which produced branches at a high frequency (Fig. 2e, f). To our knowledge, such a phenotype has not been established before in any 


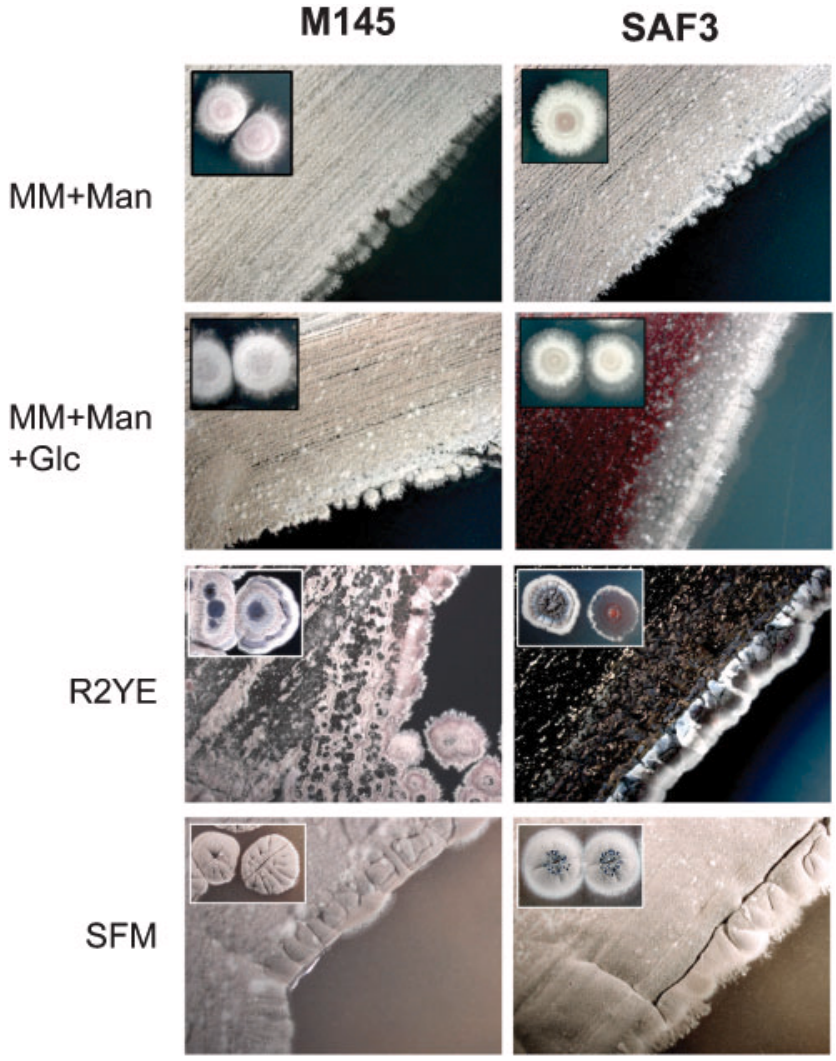

Fig. 1. Stereo light micrographs of $M 145$ and its das $A$ mutant SAF3 on different media. Strains were grown on MM with mannitol or mannitol and glucose, on R2YE and on SFM agar plates for 6 days and photographed using a Zeiss Lumar stereomicroscope. Note how glucose inhibits development inside patches of dense growth, but not around the edges. All photographed sections have a diameter of $3 \mathrm{~cm}$. The insets show representative single colonies (size of the colonies was around $5 \mathrm{~mm}$ ).

Streptomyces mutant. In contrast, under all conditions the parent M145 showed wild-type sporulation (Fig. 2a, c, e). Closer inspection by transmission electron microscopy again revealed heteromorphic spores that regularly branched (Fig. 3). Hence, the morphological defects were established experimentally by very different microscopic techniques.

Introduction of dasA on the integrative plasmid pSET151 into SAF3 reversed the defect (see Supplementary Fig. S1a, available with the online version of this paper), indicating that the inability to form aerial mycelium of the das $A$ mutant was due solely to the disruption of $\operatorname{das} A$ and that the procedure used to disrupt das $A$ has no polar effect on dasBC expression as previously reported (Saito et al., 2007).

\section{das $A$ expression is repressed by DasR and induced by chitin}

Previously, we showed that the GntR transcriptional regulator DasR was able to bind the DasR responsive
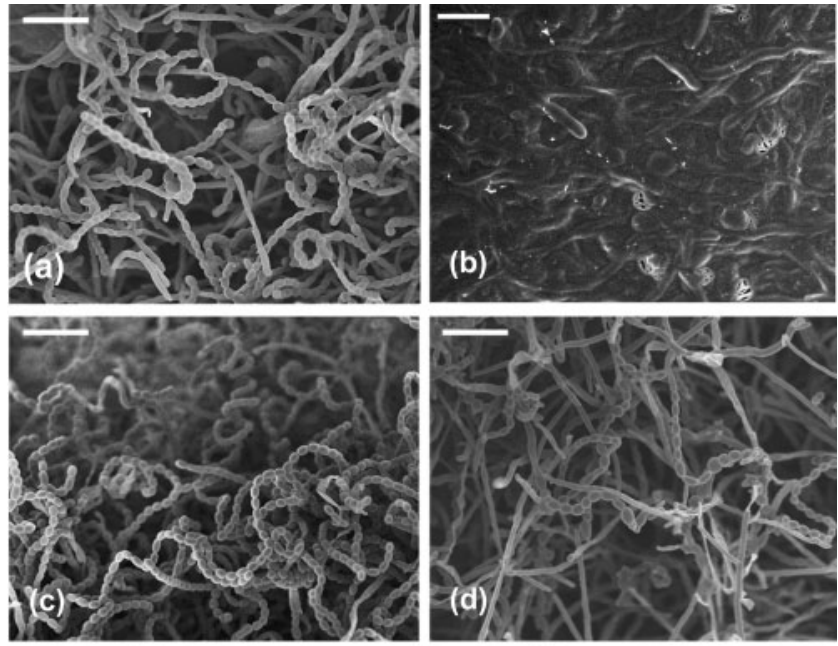

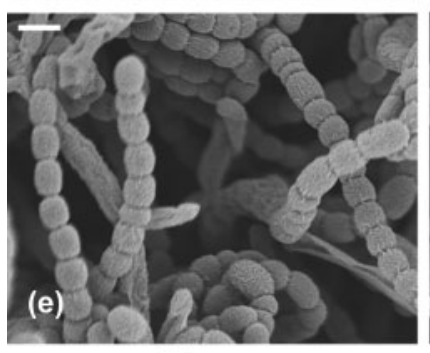

M145

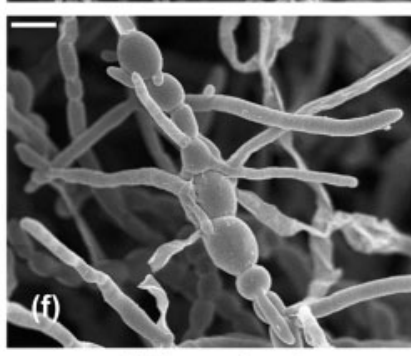

M145 $\Delta$ dasA
Fig. 2. Scanning electron micrographs of $M 145$ (a, c, e) and its $\operatorname{das} A$ mutant SAF3 (b, $d, f)$. The strains were streaked on R2YE (a, b) or SFM (c-f) agar plates, incubated for 5 days and analysed by cryo-scanning electron microscopy. On all media, S. coelicolor M145 produced abundant and wild-type spores. In contrast, on R2YE agar plates, the dasA mutant SAF3 had a bald phenotype (only vegetative growth), and on SFM agar it produced abundant aerial hyphae and spores, but the spores had many and highly unusual branches protruding from them, most likely reflecting premature germination. Scale bars: (a, c, d), $5 \mu \mathrm{m}$; (b), $2 \mu \mathrm{m} ;(\mathrm{e}, \mathrm{f})$, $1 \mu \mathrm{m}$.

element (dre) located $106 \mathrm{nt}$ upstream of the translational start of dasA (Colson et al., 2007; Rigali et al., 2004). Semiquantitative RT-PCR on RNA samples of the dasR mutant (BAP29) and the parental strain S. coelicolor M145 grown on $\mathrm{MM}+$ mannitol $(0.5 \%)$ showed the repressor role of DasR, as dasA transcription was increased about twofold throughout the life cycle in BAP29 (Fig. 4).

In order to assess the environmental context that activates dasA transcription we took advantage of the fact that the transposon integration resulted in a transcriptional fusion of the promoter of dasA (dasAp) to the egfp gene, so that the activity of dasAp can be followed by the expression of EGFP using fluorescence microscopy (Bishop et al., 2004). The dasA null mutant (SAF3) was grown on solid MM supplemented with different carbon sources and reporter gene expression was assessed (Fig. 5). The EGFP production was maximal and uniformly distributed within the 


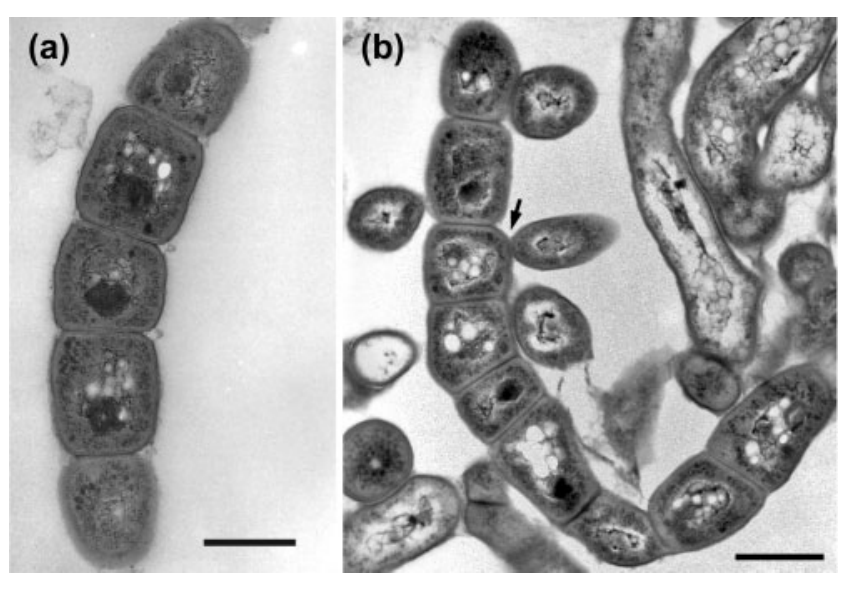

Fig. 3. High-resolution transmission electron micrographs of das $A$ mutant spore chains. Almost mature spore chains of the das $A$ mutant (b) showed several branching sites (example arrowed), most likely due to premature germination of the spores; such branching spores were also visualized by cryo-scanning electron microscopy (see Fig. 2f). Wild-type spores of the parental strain M145 (a) are shown as a control. Scale bars, $1 \mu \mathrm{m}$.

mycelia on MM supplemented with chitin as sole carbon source, while the addition of GlcNAc reduced EGFP expression to the basal level observed on $\mathrm{MM}+$ mannitol (Fig. 5). In contrast to what was previously observed (Rigali et al., 2006) and as recently reported by Saito et al. (2007), dasA is thus far the first known DasR-dependent gene that is not induced but rather repressed by GlcNAc.

To determine the relationship between DasA and chitin utilization, we compared the global chitinolytic activity of SAF3 to that of the parental strain M145 grown on MM agar plates in conditions inducing or repressing chitinase production. As a control, we generated the $\Delta$ chiSR mutant (BAP28, M145 chiS: : aacC4: : chiR; see Methods) encoding

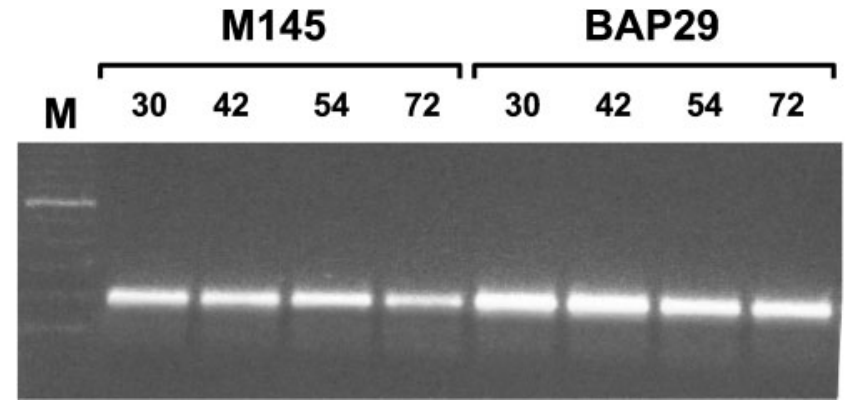

Fig. 4. $\operatorname{das} A$ is transcriptionally repressed by DasR. Transcriptional analysis of das $A$ by semiquantitative RT-PCR; RNA was collected after $30,42,54$ and $72 \mathrm{~h}$ growth on solid MM supplemented with mannitol $(0.5 \%, w / v)$. $16 \mathrm{~S}$ rRNA was used as the positive control and reactions without reverse transcriptase as the negative control (not shown). M, size markers.

the two-component system proteins ChiS/ChiR involved in the induction of the chitinolytic system in S. coelicolor (Kormanec et al., 2000). Chitinase production was not affected under repressing culture conditions, i.e. in $\mathrm{MM}+$ chitin + GlcNAc $(1 \%)$ and $\mathrm{MM}+$ chitin + glucose (1\%) (Fig. 6). Under inducing culture conditions ( $\mathrm{MM}+$ chitin), the dasA mutant showed halo sizes of chitin hydrolysis about twofold larger than those of the parental strain (Fig. 6). The increased chitinolytic and $\beta$-Nacetylglucosaminidase activities were assessed by colorimetric assays (see Methods), and were estimated to be about $30 \%$ higher than those of the wild-type strain after $48 \mathrm{~h}$ growth at $28{ }^{\circ} \mathrm{C}$. Complementation of SAF3 with dasA cloned into pSET151 restored a normal chitinolytic activity, indicating that overproduction of chitinases in the das $A$ mutant was due solely to the disruption of das $A$ (see Supplementary Fig. S1b). A model which explains the role of DasA in the induction of the chitinolytic system is presented in the Discussion.
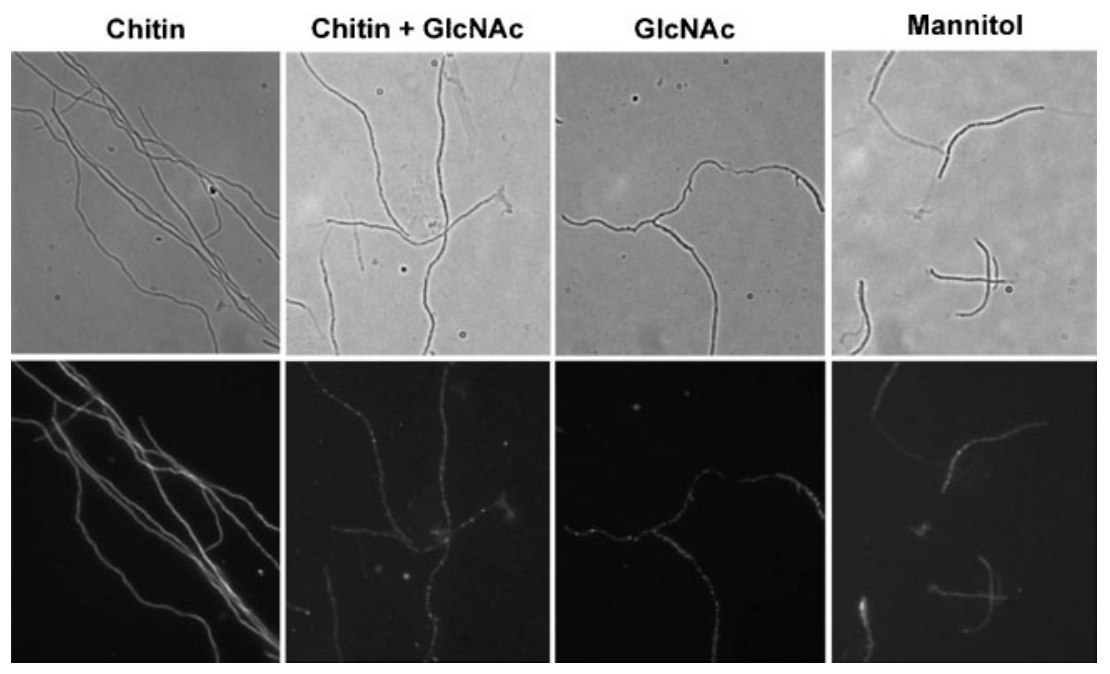

Fig. 5. das $A$ is induced by chitin. Transmission and fluorescence images from confocal microscopy are shown at the top and bottom respectively. The expression of the EGFP protein is under the control of the das $A$ promoter in the SAF3 mutant. EGFP production is maximal and uniformly distributed within the mycelia of SAF3 grown on MM supplemented with chitin (1\%) as sole carbon source. No differences in the levels of fluorescence were observed on chitin + GlcNAc, GlcNAc ( $1 \%)$ or mannitol (0.5\%). 


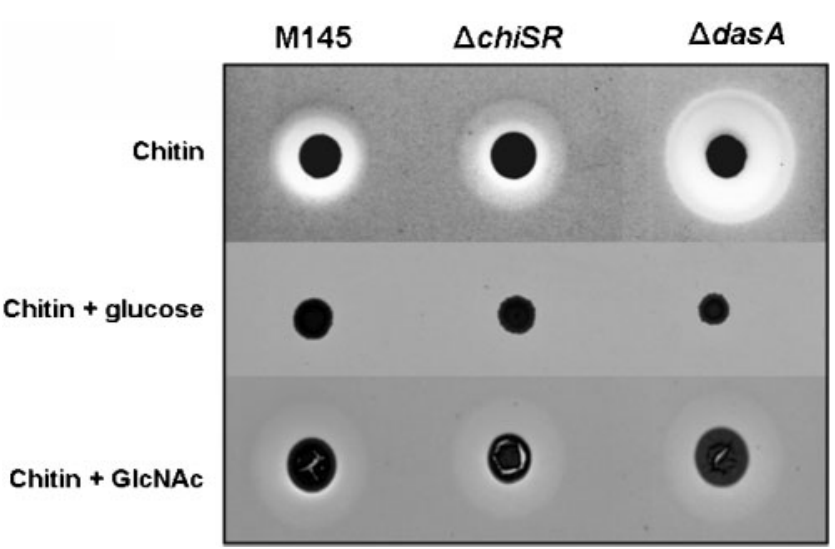

Fig. 6. Global chitinolytic activity of the $\operatorname{das} A$ mutant. Spore suspensions of $S$. coelicolor M145 (parental strain), $\Delta$ chiSR (BAP28) and $\triangle$ dasA (SAF3) were spotted on MM inducing (chitin) or repressing (chitin + GlcNAc or chitin + glucose) the chitinolytic system. The chitinolytic activity is visualized by the haloes around the colonies. All sugars were added to a concentration of $1 \%(\mathrm{w} /$ v). M145 and the $\Delta$ chiSR mutant were used as positive and negative controls, respectively.

\section{Chitin and the control of development in streptomycetes}

The bald phenotype of both dasR and dasA mutants suggested an association between nutrient utilization - specifically of monomer and polymer forms of GlcNAc and development. This correlation was previously demonstrated with GlcNAc, as R2YE agar plates containing GlcNAc $(0.5 \% \mathrm{w} / \mathrm{v})$ blocked development of $S$. coelicolor M145. Here we show that the 'GlcNAc effect' is widespread but not universal for streptomycetes, as the effect was observed for most but not all streptomycetes tested so far (Fig. 7). This effect was explained by the model presented previously (Fig. 8) (Rigali et al., 2006): when the extracellular concentration of GlcNAc is high, the components of the phosphoenolpyruvate (PEP)-dependent phosphotransferase system (PTS) would actively transfer the phosphate group from PEP to GlcNAc, preventing the PTS-mediated control of development-specific proteins, which would therefore be unable to trigger formation of aerial hyphae. Without GlcNAc, the phosphate group from PEP is transferred to one or more as yet unidentified developmental proteins, resulting in development on rich media (such as R2YE agar). This model suggests that GlcNAc possesses at least an equivalent position in the utilization hierarchy to glucose, which is the main carbon source in R2YE agar.

We assessed whether GlcNAc originating from chitin would have the same inhibiting effect on development. In the presence of chitin, GlcNAc is derived from the hydrolysis of chitobiose (notably via the $\beta$ - $N$-acetylglucosaminidase DasD) and is phosphorylated via NagK (the GlcNAc kinase) and not by the PTS (Fig. 8). Moreover, as glucose in R2YE exerts a strong carbon catabolite

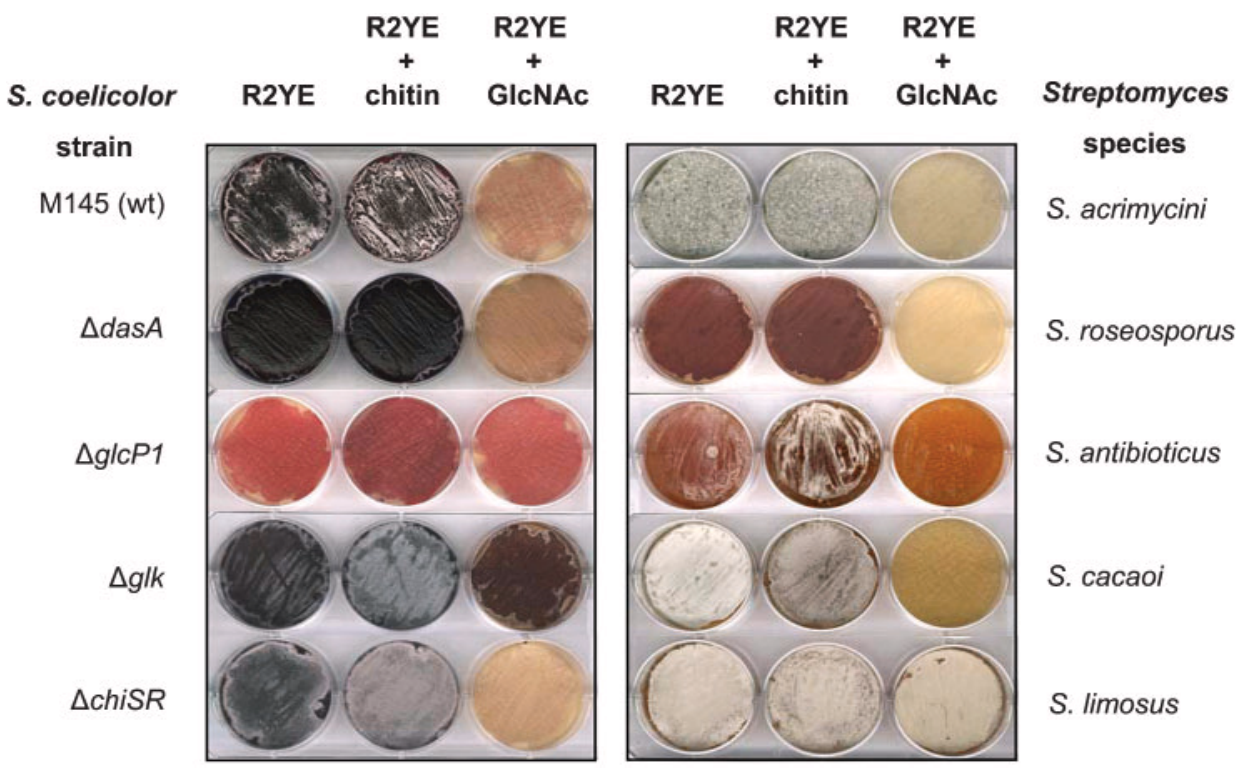

Fig. 7. Effect of GlcNAc and chitin on development in S. coelicolor M145 and other streptomycetes on R2YE medium. Null mutants for the glucose transporter (M145 $\Delta g / c P 1)$ and for glucose kinase (M145 $\Delta g / k$ ) were used as controls to monitor the effect of chitin and GlcNAc on R2YE plates without the effect of glucose repression. While M145 develops normally on R2YE, concentrations of GlcNAc above $10 \mathrm{mM}$ (20 mM is shown) inhibit development and antibiotic production - particularly that of the blue-pigmented actinorhodin. $\triangle c h i S R$ and $\Delta$ das $A$ mutants, defective and improved for chitin utilization respectively, were used as negative and positive control to monitor the effect of chitin on morphological differentiation. 

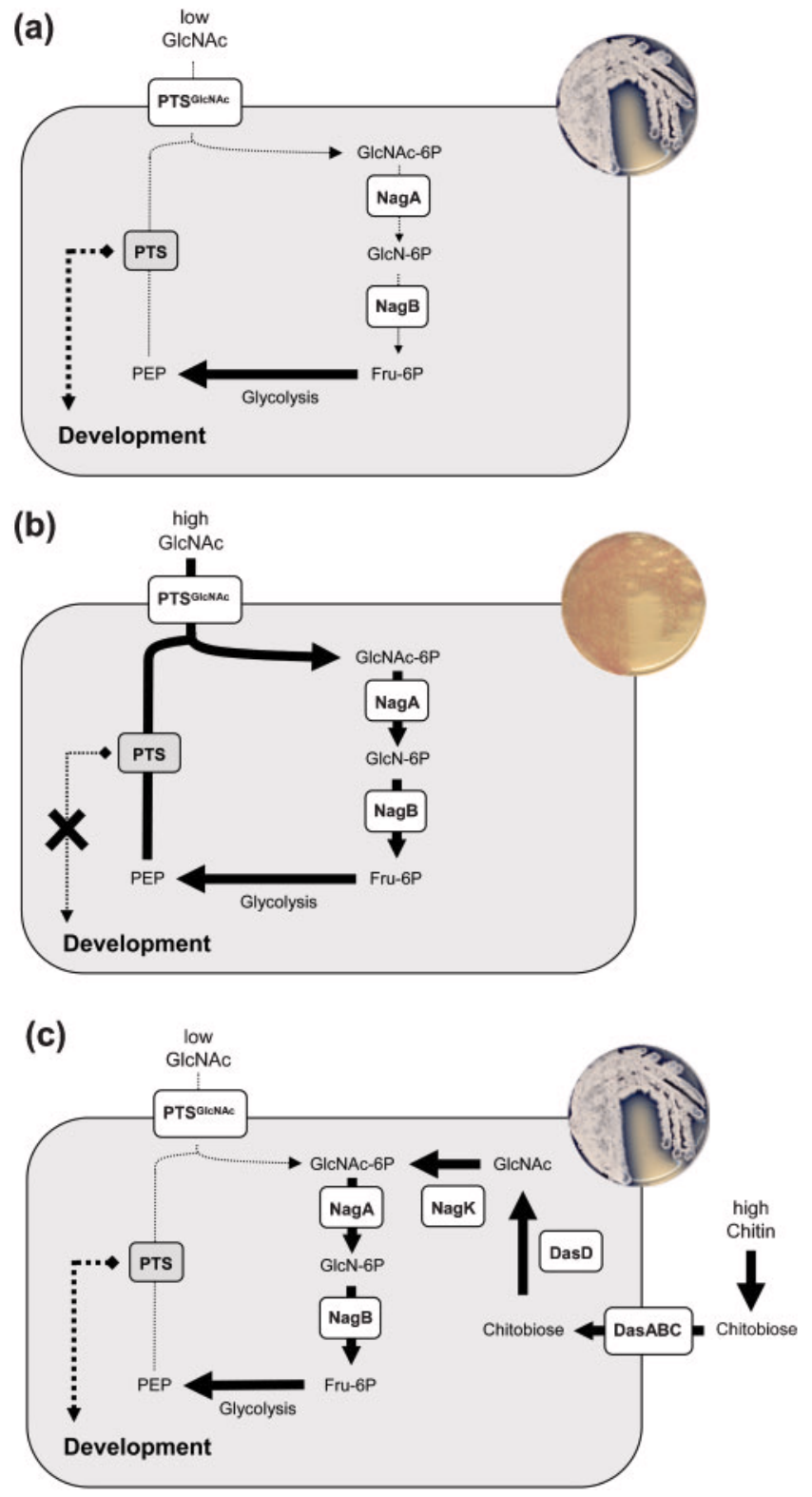

repression on chitin utilization, the availability of GlcNAc from chitin is significantly delayed. Therefore, as expected, for all streptomycetes tested so far, the addition of chitin into R2YE is not able to block development, and for some strains sporulation is stimulated and/or occurs precociously (Fig. 7). In order to check the 'chitin effect' on development of $S$. coelicolor in strains insensitive to glucose catabolite repression (Angell et al., 1994), we streaked the glucose transporter mutant BAP17 $(\Delta g l c P 1)$, and a new glucose kinase null mutant of $S$. coelicolor (M145 $\Delta$ glkA; B. Traag, S. Colson, S. Rigali \& G. P. van Wezel, unpublished) on R2YE, R2YE + chitin and R2YE + GlcNAc. The glcP1 mutant BAP17 showed a bald phenotype on all media and, interestingly, the 'GlcNAc effect' was lost, as BAP17 was insensitive to a high concentration of GlcNAc, which blocks development of S. coelicolor M145 (Fig. 7). The fact
Fig. 8. Effects of addition of GlcNAc or chitin on development of S. coelicolor grown in R2YE medium. On R2YE (a), the PTS GlcNAc is expressed at its basal level. Under these conditions the intracellular PTS components would transfer the phosphate from PEP to PTS development-specific target proteins. Upon addition of GlcNAc (b), GlcNAc is transported and metabolized to glucosamine 6-phosphate (GlcN-6P) and this then induces the GlcNAc regulon by inhibiting DasR's DNA-binding ability (Rigali et al., 2006). Under these conditions, the PTS components actively transfer the phosphate from PEP to GlcNAc, preventing the PTSmediated control of development-specific proteins. Upon addition of chitin in R2YE (c), GlcNAc is derived from the hydrolysis of chitobiose (notably via the $\beta-N$-acetylglucosaminidase DasD) and is phosphorylated via NagK (the GlcNAc kinase) and not by the PTS. The phosphate from PEP is not sequestrated by the incoming sugar and PTS protein can target developmental proteins for the phosphotransfer. Abbreviations: NagK, GlcNAc kinase; DasD, $\beta$ $N$-acetylglucosaminidase; DasABC, the chitobiose transporter; NagA, GlcNAc-6P deacetylase; NagB, GlcN-6P isomerase.

that in both the presence and the absence of glucose repression (i.e. in M145 and in M145 $\Delta g l k A$, respectively) sporulation was normal on R2YE + chitin strongly suggests that the failure of chitin to inhibit or delay development of $S$. coelicolor is not due to glucose repression of the chitinolytic system. Therefore, GlcNAc originating from chitin, which enters the cell not via the PTS but via the PTS-independent DasABC transporter, does not deliver the same information to the developmental programme (Fig. 8). Interestingly, the GlcNAc blocking effect was less severe for mutants affected in glucose repression, i.e. in $\triangle g l k A$ and $\Delta g l c P 1$ (Fig. 7), suggesting signalling connections between glucose and GlcNAc-related processes.

\section{DISCUSSION}

We show here that the DasABC chitobiose transporter is a second DasR-dependent sugar transporter, in addition to the PTS ${ }^{\mathrm{GlcNAc}}$, that plays an important role in both primary metabolism and morphological differentiation of $S$. coelicolor. Regarding the role of DasA in primary metabolism, the increased global chitinolytic activity observed for the dasA mutant may seem unusual, as inactivation of the chitobiose sensory protein should rather prevent the extracellular sensing of chitin as well as the transport of the chitin-derived inducer of the chitinolytic system. Expression of the chitinolytic genes is rigorously regulated and a plausible explanation for the role of DasA function is inferred from the model of the induction of the chitinolytic system in Vibrio species (Li \& Roseman, 2004). This model (Fig. 9) comprises three components: (i) the environmental signal, (GlcNAc) ${ }_{2}$, and possibly larger chitin oligosaccharides; (ii) DasA, the solute-binding protein specific for $(\mathrm{GlcNAc})_{n}(n>1)$, and its respective ABC-type permeases DasB and DasC; and (iii) the two-component regulatory system ChiS/ChiR. Disruption of $c h i R$ caused reduced expression of chiC in S. coelicolor (Kormanec et al., 


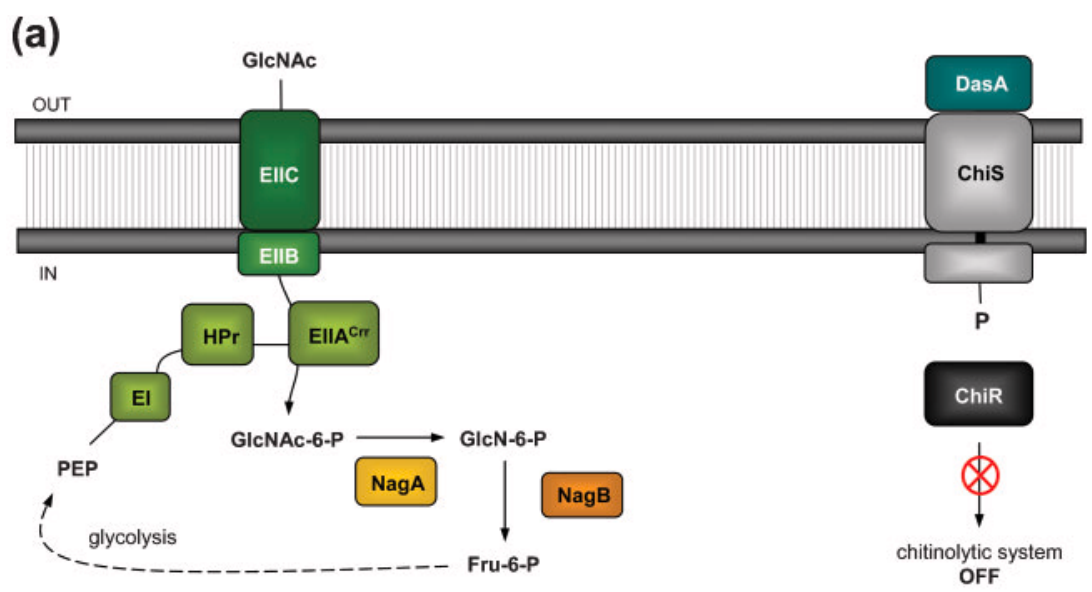

Fig. 9. Model for the regulation of the chitinolytic system in $S$. coelicolor. (a) Repression ('minus' phenotype) and (b) induction ('plus' phenotype) of the chitinolytic system in S. coelicolor. In the presence of chitobiose or a related metabolic product of chitin, ChiS is autophosphorylated at a conserved histidine residue ( $\mathrm{H} 1199)$. The phosphoryl group is then transferred to a conserved aspartate (D54) of ChiR, which then binds to the chi promoter regions to activate expression of chitinolytic (chi) genes. The repression of chigenes, the 'minus' phenotype, would be the result of binding of DasA to the extracellular domain of the sensor ChiS, 'locking' it into an inactive conformation. In the presence of chitin, the extracellular signal, typically (GlcNAc) ${ }_{2}$, competes with the sensor ChiS for binding to DasA. ChiS could not bind the DasA$(\mathrm{GlcNAc})_{2}$ complex and would thereby be activated to the 'plus' phenotype, followed by the transcriptional activation of the chitinolytic genes by ChiR.

2000). The suggested model predicts that in the presence of chitobiose or a related chitin breakdown product, ChiS is autophosphorylated at a conserved histidine residue (H1199). The phosphoryl group is then transferred to a conserved aspartate (D54) of ChiR, which then binds to the chi promoter regions to activate expression of chi genes. According to what is proposed in Vibrio (Li \& Roseman, 2004), the repression of the chitinolytic genes, the 'minus' phenotype, would be the result of the binding of DasA to the extracellular domain of the sensor ChiS, thereby 'locking' it into an inactive conformation (Fig. 9a). In the presence of chitin, the extracellularly generated $(\mathrm{GlcNAc})_{n}$ or, more specifically, (GlcNAc) ${ }_{2}$, competes with the sensory ChiS for DasA. ChiS could not bind the DasA-(GlcNAc) $)_{2}$ complex and would be thereby activated to the 'plus' phenotype (Fig. 9b), whereafter the chitinolytic genes are expressed via the activation of ChiR. According to this model, the absence of DasA (i.e. in the dasA null mutant) would leave the sensor ChiS permanently in its activated form, leading to high and constitutive production of chitinases.

From the developmental point of view, the das $A$ mutant has an extraordinary and unique phenotype in that spore chains show extensive germination, a phenomenon that has to our knowledge never been described before. This premature germination could well be a secondary effect of the disorder of GlcNAc utilization in dasA mutants, and in particular due to a lack of the peptidoglycan precursor molecule glucosamine 6-phosphate. It is easy to see how lower levels of this compound could lead to weakened cell walls and therefore premature germination. It is as yet unclear why the inactivation of the chitobiose transporter causes developmental arrest of $S$. coelicolor, and especially when grown in the absence of chitin or chitobiose. GlcNAc (transported by the PTS) and chitobiose (transported by DasABC) are both degradation products of the GlcNAc polymer chitin. However, in contrast to GlcNAc, chitin does not block development of streptomycetes (Fig. 7). Considering the glucose-dependent developmental defects of dasA mutants (Fig. 1), it will be important to see whether glucose utilization is affected. As shown in this paper, deletion of das $A$ causes an increase in the total chitinolytic activity. One possibility is that the absence of DasA simulates the abundance of the alternative rich carbon/nitrogen source chitin in the environment, and, via an unknown mechanism, prevents the utilization of more 
readily metabolized carbon sources such as glucose, resulting in poor growth and lack of development on glucosecontaining media. We have strong evidence for intimately correlated connections between glucose, GlcNAc and chitin utilization pathways in S. coelicolor. For instance, preventing GlcNAc or chitin utilization counteracts the induction of glucose transport (H. Nothaft \& F. Titgemeyer, unpublished data). Additionally, the inactivation of dasR, which controls both GlcNAc and chitin utilization, results in enhanced glucose catabolite repression (S. Rigali, G. P. van Wezel \& F. Titgemeyer, unpublished data). In our opinion, an insight into the regulatory pathways that govern access to glucose or GlcNAc utilization is pivotal in understanding the complex mechanisms that relay information about the state of primary metabolism to morphogenesis in streptomycetes.

The DasA/DasR/PTS/ChiSR system could well be much more complicated, with additional elements participating in the model. In fact, the bald phenotype of dasA mutants is primarily observed in densely populated sections, while well-separated single colonies sporulate relatively normally, suggesting that the phenotype of these mutants is controlled by a quorum-sensing-like mechanism. In Gram-positive bacteria, quorum sensing often involves regulatory proteins belonging to the two-component system group (Kleerebezem et al., 1997). Conceivably, the ChiS/ChiR system predicted to interact with DasA could also target one or more developmental genes whose expression is cell density-dependent.

DasA is not the first example of an ABC transporter solutebinding protein that is also involved in the control of morphological differentiation of streptomycetes. This is exemplified by the bldK operon, which encodes an oligopeptide importer responsible for the import of an early extracellular signalling molecule for cell differentiation (Nodwell et al., 1996). The reverse is also known, with deletion of the gene for the phosphate-binding protein PstS accelerating (rather than inhibiting) development of Streptomyces lividans (Diaz et al., 2005). Additionally, a study aimed at isolating morphogenesis-dependent ADPribosylated proteins exclusively identified ligand-binding elements of $\mathrm{ABC}$ transporters in S. coelicolor (Sugawara et al., 2002). Post-translational modifications such as ADPribosylation can prevent the export of these proteins to the surface and therefore inhibit their function in transport and development. Interestingly, the proteomic analysis of the germination-deficient $\operatorname{crp}$ mutant $(\Delta \mathrm{SCO} 3571)$ revealed that several $\mathrm{ABC}$ transporter solute-binding proteins were sequestered intracellularly, perhaps disturbing the perception of the environmental signals that trigger germination (Piette et al., 2005; Derouaux et al., 2004a, b). Thus, it is becoming increasingly clear that extracellular solute-binding proteins such as DasA play an important role in the perception of nutritional signals that control the onset of key morphological processes.

The relationship between the chitobiose sensor/transporter DasA and development suggests that the deletion of
ABC-type sensors/transporters of other abundant carbon sources like cellobiose (CebE) and xylobiose (BxlE) would lead to similar repercussions on morphological differentiation. The same could apply to other sugar transporters predicted to be part of the DasR regulon; for example, highly significant potential DasR responsive elements have been predicted upstream of SCO4286, SCO6005 and SCO2946, all encoding sugar-binding components of ABC-type transporters (Hiard et al., 2007; Colson et al., 2007). Mutational analysis of these and other sugar transporters is currently under way and the outcome of these experiments should provide more insight into the close link between sugar utilization and the onset of development.

\section{ACKNOWLEDGEMENTS}

The Belgian government supported this work as part of the Interuniversity Poles of Attraction Programme (PAI P5/33). H.N. and F.T. were supported through grant SFB473 of the Deutsche Forschungsgemeinschaft. S. C. is a research fellow of the Fund of Research in Industry and Agriculture (FRIA) and S. R. was supported by the patrimoine of the University of Liège (Belgium). We would like to thank Bjørn Traag for unpublished data, and Lorena Fernandez and Paul Dyson for the gift of the dasA knock-out cosmid.

\section{REFERENCES}

Angell, S., Lewis, C. G., Buttner, M. J. \& Bibb, M. J. (1994). Glucose repression in Streptomyces coelicolor A3(2): a likely regulatory role for glucose kinase. Mol Gen Genet 244, 135-143.

Bishop, A., Fielding, S., Dyson, P. \& Herron, P. (2004). Systematic insertional mutagenesis of a streptomycete genome: a link between osmoadaptation and antibiotic production. Genome Res 14, 893-900.

Chater, K. F. (1972). A morphological and genetic mapping study of white colony mutants of Streptomyces coelicolor. J Gen Microbiol 72, 9-28.

Chater, K. F. \& Horinouchi, S. (2003). Signalling early developmental events in two highly diverged Streptomyces species. Mol Microbiol 48, 9-15.

Colson, S., Stephan, J., Hertrich, T., Saito, A., van Wezel, G. P., Titgemeyer, F. \& Rigali, S. (2007). Conserved cis-acting elements upstream of genes composing the chitinolytic system of streptomycetes are DasR-responsive elements. J Mol Microbiol Biotechnol 12, 60-66.

Derouaux, A., Dehareng, D., Lecocq, E., Halici, S., Nothaft, H., Giannotta, F., Moutzourelis, G., Dusart, J., Devreese, B. \& other authors (2004a). Crp of Streptomyces coelicolor is the third transcription factor of the large CRP-FNR superfamily able to bind cAMP. Biochem Biophys Res Commun 325, 983-990.

Derouaux, A., Halici, S., Nothaft, H., Neutelings, T., Moutzourelis, G., Dusart, J., Titgemeyer, F. \& Rigali, S. (2004b). Deletion of a cyclic AMP receptor protein homologue diminishes germination and affects morphological development of Streptomyces coelicolor. J Bacteriol 186, 1893-1897.

Diaz, M., Esteban, A., Fernandez-Abalos, J. M. \& Santamaria, R. I. (2005). The high-affinity phosphate-binding protein PstS is accumulated under high fructose concentrations and mutation of the corresponding gene affects differentiation in Streptomyces lividans. Microbiology 151, 2583-2592. 
Godden, B., Legon, T., Helvenstein, P. \& Penninckx, M. (1989). Regulation of the production of hemicellulolytic and cellulolytic enzymes by a Streptomyces sp. growing on lignocellulose. J Gen Microbiol 135, 285-292.

Hiard, S., Maree, R., Colson, S., Hoskisson, P. A., Titgemeyer, F., van Wezel, G. P., Joris, B., Wehenkel, L. \& Rigali, S. (2007). PREDetector: a new tool to identify regulatory elements in bacterial genomes. Biochem Biophys Res Commun 357, 861-864.

Hillerich, B. \& Westpheling, J. (2006). A new GntR family transcriptional regulator in Streptomyces coelicolor is required for morphogenesis and antibiotic production and controls transcription of an $\mathrm{ABC}$ transporter in response to carbon source. J Bacteriol 188, 7477-7487.

Hopwood, D. A., Wildermuth, H. \& Palmer, H. M. (1970). Mutants of Streptomyces coelicolor defective in sporulation. J Gen Microbiol 61, 397-408.

Hoskisson, P. A., Rigali, S., Fowler, K., Findlay, K. C. \& Buttner, M. J. (2006). DevA, a GntR-like transcriptional regulator required for development in Streptomyces coelicolor. J Bacteriol 188, 5014-5023.

Kieser, T., Bibb, M. J., Buttner, M. J., Chater, K. F. \& Hopwood, D. A. (2000). Practical Streptomyces Genetics. Norwich, UK: John Innes Foundation.

Kleerebezem, M., Quadri, L. E., Kuipers, O. P. \& de Vos, W. M. (1997). Quorum sensing by peptide pheromones and two-component signal-transduction systems in Gram-positive bacteria. Mol Microbiol 24, 895-904.

Kormanec, J., Sevcikova, B. \& Homerova, D. (2000). Cloning of a two-component regulatory system probably involved in the regulation of chitinase in Streptomyces coelicolor A3(2). Folia Microbiol (Praha) 45, 397-406.

Kubota, T., Miyamoto, K., Yasuda, M., Inamori, Y. \& Tsujibo, H. (2004). Molecular characterization of an intracellular $\beta$-N-acetylglucosaminidase involved in the chitin degradation system of Streptomyces thermoviolaceus OPC-520. Biosci Biotechnol Biochem 68, 1306-1314.

Li, X. \& Roseman, S. (2004). The chitinolytic cascade in Vibrios is regulated by chitin oligosaccharides and a two-component chitin catabolic sensor/kinase. Proc Natl Acad Sci U S A 101, 627-631.

Miyashita, K., Fujii, T. \& Saito, A. (2000). Induction and repression of a Streptomyces lividans chitinase gene promoter in response to various carbon sources. Biosci Biotechnol Biochem 64, 39-43.

Nodwell, J. R., McGovern, K. \& Losick, R. (1996). An oligopeptide permease responsible for the import of an extracellular signal governing aerial mycelium formation in Streptomyces coelicolor. Mol Microbiol 22, 881-893.

Nodwell, J. R., Yang, M., Kuo, D. \& Losick, R. (1999). Extracellular complementation and the identification of additional genes involved in aerial mycelium formation in Streptomyces coelicolor. Genetics 151, $569-584$.

Nothaft, H., Dresel, D., Willimek, A., Mahr, K., Niederweis, M. \& Titgemeyer, F. (2003). The phosphotransferase system of Streptomyces coelicolor is biased for $\mathrm{N}$-acetylglucosamine metabolism. J Bacteriol 185, 7019-7023.

Piette, A., Derouaux, A., Gerkens, P., Noens, E. E., Mazzucchelli, G., Vion, S., Koerten, H. K., Titgemeyer, F., De Pauw, E. \& other authors (2005). From dormant to germinating spores of Streptomyces coelicolor A3(2): new perspectives from the crp null mutant. J Proteome Res 4, 1699-1708.

Pope, M. K., Green, B. D. \& Westpheling, J. (1996). The bld mutants of Streptomyces coelicolor are defective in the regulation of carbon utilization, morphogenesis and cell-cell signalling. Mol Microbiol 19, 747-756.

Rigali, S., Derouaux, A., Giannotta, F. \& Dusart, J. (2002). Subdivision of the helix-turn-helix GntR family of bacterial regulators in the FadR, HutC, MocR, and YtrA subfamilies. J Biol Chem 277, 12507-12515.

Rigali, S., Schlicht, M., Hoskisson, P., Nothaft, H., Merzbacher, M., Joris, B. \& Titgemeyer, F. (2004). Extending the classification of bacterial transcription factors beyond the helix-turn-helix motif as an alternative approach to discover new cis/trans relationships. Nucleic Acids Res 32, 3418-3426.

Rigali, S., Nothaft, H., Noens, E. E., Schlicht, M., Colson, S., Muller, M., Joris, B., Koerten, H. K., Hopwood, D. A. \& other authors (2006). The sugar phosphotransferase system of Streptomyces coelicolor is regulated by the GntR-family regulator DasR and links $N$-acetylglucosamine metabolism to the control of development. Mol Microbiol 61, 1237-1251.

Ryding, N. J., Kelemen, G. H., Whatling, C. A., Flardh, K., Buttner, M. J. \& Chater, K. F. (1998). A developmentally regulated gene encoding a repressor-like protein is essential for sporulation in Streptomyces coelicolor A3(2). Mol Microbiol 29, 343-357.

Saito, A., Shinya, T., Miyamoto, K., Yokoyama, T., Kaku, H., Minami, E., Shibuya, N., Tsujibo, H., Nagata, Y. \& other authors (2007). The $\operatorname{das} A B C$ gene cluster, adjacent to dasR, encodes a novel ABC transporter for the uptake of $N, N^{\prime}$-diacetylchitobiose in Streptomyces coelicolor A3(2). Appl Environ Microbiol 73, 3000-3008.

Seo, J. W., Ohnishi, Y., Hirata, A. \& Horinouchi, S. (2002). ATPbinding cassette transport system involved in regulation of morphological differentiation in response to glucose in Streptomyces griseus. J Bacteriol 184, 91-103.

Sugawara, K., Dohmae, N., Kasai, K., Saido-Sakanaka, H., Okamoto, S., Takio, K. \& Ochi, K. (2002). Isolation and identification of novel ADP-ribosylated proteins from Streptomyces coelicolor A3(2). Biosci Biotechnol Biochem 66, 2292-2296.

van Wezel, G. P., Mahr, K., Konig, M., Traag, B. A., Pimentel-Schmitt, E. F., Willimek, A. \& Titgemeyer, F. (2005). GlcP constitutes the major glucose uptake system of Streptomyces coelicolor A3(2). Mol Microbiol 55, 624-636.

Zhang, H., Huang, X., Fukamizo, T., Muthukrishnan, S. \& Kramer, K. J. (2002). Site-directed mutagenesis and functional analysis of an active site tryptophan of insect chitinase. Insect Biochem Mol Biol 32, 1477-1488.

Edited by: P. R. Herron 